Xiaoqiao $\mathrm{Wu}^{*}$

\title{
Neue Quellenfunde zur frühen Rezeption der Kinder- und Hausmärchen der Brüder Grimm im China der ersten zwei Jahrzehnte des 20. Jahrhunderts Findings of New Sources of the Early Reception of Children's and Household Tales of the Brothers Grimm in China in the First Two Decades of the 20th Century
}

https://doi.org/10.1515/ifdck-2021-2005

Zusammenfassung: Die frühe chinesische Rezeption der Kinder- und Hausmärchen (KHM) der Brüder Grimm, die in das letzte Jahrzehnt der Qing-Dynastie fällt und bis zur 4. Mai-Bewegung 1919 (五四运动) dauerte, zeichnet sich durch eine auffallende Anonymität aus. Wegen der Ausblendung von Autoren- und unter Umständen zumeist auch von Übersetzernamen sowie verwirrenden Variationen bei Angabe der Gattungsbezeichnung Märchen fällt es bisherigen Forschern schwer, einschlägige Übersetzungs- und Rezeptionsbelege zu identifizieren. Der vorliegende Aufsatz unternimmt den Versuch, mittels neu recherchierter Befunde die Desiderate in der bisherigen Forschung zu beseitigen. Es wird ein besonderes Augenmerk gerichtet auf die in der bisherigen Forschung kaum beachtete originale Vorlage der ersten chinesischen KHM-Sammlung Shixie sowie auf einige zerstreut in Zeitschriften und Zeitungen publizierte Belegtexte, die hier zum ersten Mal zur Kenntnis genommen werden.

Article Note: Der vorliegende Beitrag ist im Rahmen eines von dem National Social Science Fund of China geförderten Projekts (2015年度国家社科基金年度项目《晚清民初稀见德语文学 译作考》) (Projektnummer: 15BWW057) zur Erschließung von entlegenen chinesischen Übersetzungs- und Rezeptionszeugnissen der deutschsprachigen Literatur im Zeitraum von der Spätqing-Dynastie bis zu den ersten Jahrzehnten der Bürgerlichen Republik entstanden. Ich bedanke mich bei Prof. Dr. Alfred Messerli (Zürich) für seine freundlichen Anregungen.

*Korrespondenzautor: Prof. Dr. Xiaoqiao Wu, Department of German, Beihang University, Xueyuan Road 37 Haidian District, 100191 Beijing, China. E-Mail: xiaoqiaowu@hotmail.com 
Stichwörter: Kinder- und Hausmärchen der Brüder Grimm, Geschichte der chinesische Rezeption der deutschen Literatur, Quellenforschung

\begin{abstract}
The early reception of Children's and Household Tales (CHT) of the Brothers Grimm in China, which took place in the last decade of the Qing Dynasty and lasted until the May 4th Movement in 1919, is characterized by a distinct anonymity. Due to the absence of the names of the authors and, if necessary, the names of the translators as well as the confusing variants when specifying the generic term fairy tales, previous researchers have found it difficult to identify relevant evidence of translation and reception of $C H T$. The present paper attempts to fulfill the deficiency in previous research on the basis of findings in new studies. Particular attention is paid to the original manuscript of the first Chinese CHT-collection Shixie, which has hardly been noticed in previous research, as well as to a few proof texts published in scattered magazines and newspapers, which have been noted here for the first time.
\end{abstract}

Keywords: Children's and Household Tales, history of the Chinese reception of the German literature, research of new sources.

\title{
1 Einleitung
}

Gab es in der Qing-Dynastie, die 1911 wegen der Xinhai-Revolution (辛亥革 命) abdanken musste, bereits chinesische Übersetzungen von den Kinder- und Hausmärchen - im Folgenden als KHM abgekürzt - der Brüder Grimm? Wenn ja, welche KHM-Titel wurden von wem übersetzt? Wo erschienen die Texte? Welcher Vorlagen bedienten sich die Übersetzungen? Seit den 1980er Jahren versuchte sich die internationale Forschung bei der Untersuchung der frühen chinesischen Rezeption der KHM mit großem Interesse solchen Fragestellungen zu widmen.

Das Wissen über die frühe Rezeption von Grimms Märchen in China fand de facto im Zuge der Verbreitung der Märchenforschung und -übersetzung nach der 4. Mai-Bewegung 1919 nach und nach vermehrt Beachtung. Schon der renommierte Märchenforscher und -übersetzer Zhao Jingshen (赵景深 19021985) ging in den 1920er Jahren den ersten Spuren der KHM im chinesischen Schrifttum nach. Zhao konnte damals allerdings nur wenige Übersetzungs- und Rezeptionsbelege ermitteln und nahm die meisten $K H M$-Übersetzungen in dem ersten Jahrzehnt des 20. Jahrhunderts nicht zur Kenntnis (vgl. Zhao 1924 [1922]: 183-186). Erst Anfang der 1980er Jahre begann sich die Forschung bewusst und systematisch mit der Untersuchung der frühen Verbreitung und Rezeption der 
KHM im chinesischen Schrifttum zu befassen. So machte der chinesische Forscher Hu Congjing (胡从经) 1982 auf die in der Forschung kaum beachtete Verbreitung der ausländischen Kinderliteratur in der Spät-Qing-Dynastie aufmerksam und gab als Erster konkrete Hinweise auf die frühe Übertragung der KHM durch den namhaften Übersetzer Zhou Guisheng (周桂笙) und dessen 1903 in Shanghai erschienenes Buch Xin'an xieyi chubian (新庵谐译初编 Erstmals gesammelte von Xin An angefertigte Übersetzungen von scherzhaften Texten) (Hu 1982: 148-159). Hu konnte damals allerdings nur ein einziges Märchen von Grimm, im Konkreten Hamo taizi (蛤蟆太子 Der Froschkönig oder der eiserne Heinrich), deutlich identifizieren, obwohl er zugleich noch die Übersetzung von Lang yang fuchou (狼羊复仇 Der Wolf und die sieben jungen Geißlein), einem weiteren Text aus den Kinder- und Hausmärchen, wiedergab (vgl. Hu 1982: 150151, 154-159). Seine These, der zweite Band der Xin'an xieyi chubian von Zhou Guisheng enthalte die Übersetzungen aus den Fabeln Äsops, Wilhelm Hauffs Märchen und Grimms Märchen, wurde von der frühen Forschung zur Rezeption der Brüder Grimm in China lange Zeit vertreten. Inzwischen sind drei Dissertationen zur Rezeption der Brüder Grimm in China erschienen: Liang Yea-Jen (梁 雅贞) schloss 1986 ihre Bamberger Dissertation Kinder- und Hausmärchen der Brüder Grimm in China: Rezeption und Wirkung ab (Liang 1986). Fu Pinjing (付品 晶) promovierte 2010 mit der Arbeit unter dem Titel Gelin tonghua zai zhongguo (格林童话在中国 KHM in China) in Chengdu (Fu 2010). Xia Lu (陆霞) publizierte ihre 2011 fertiggestellte Aachener Dissertation Grimms Märchen in China: Begegnung, Rezeption und Wirkung sowohl in Deutschland (Lu 2011) als auch in China (Lu 2012). In allen Arbeiten sind bei den Darstellungen der frühen Rezeption von Grimms Kinder- und Hausmärchen in China Fehler unterlaufen, die in den folgenden Ausführungen noch zu erörtern sind. Anlässlich des 200-jährigen Jubiläums der KHM erschienen eine Reihe Aufsätze zur Rezeption der KHM der Brüder Grimm in China. Was die Quelle von Zhou Guisheng betrifft, haben die meisten Forscher die teilweise bereits überholten Forschungsergebnisse der Untersuchungen von Hu Congjing (1982) und Wu Hongyu (伍红玉) (2006) einfach übernommen (z. B. Du 2013: 268-269). Auch der Beitrag von Dechao Li erwähnte bei der Übersichtsdarstellung der chinesischen Übersetzung der KHM am Anfang des 20. Jahrhunderts zwar Zhou Guishengs frühe chinesische Übersetzung in dessen Sammlung Xin'an xieyi chubian, aber konnte in Anlehnung an die Untersuchung von Wu Hongyu (2010: 156) lediglich sechs Märchen davon identifizieren (vgl. Li 2014: 122). 


\section{Grimms Märchen in der Sammlung Xin’an xieyi chubian}

Tonghua (童话), die bisherige geläufige chinesische Übertragung des deutschen Begriffes Märchen, tauchte, soviel wir wissen, erst 1909 auf, als Sun Yuxiu (孙 毓修 1871-1922) in Shanghai mit dem Vorhaben begann, seine berühmte Reihe Märchen herauszugeben (vgl. Sun 2013: 131). Heute hält die Forschung daran fest, dass die Märchen der Brüder Grimm bereits in der Spät-Qing-Dynastie ins Chinesische übertragen wurden. In den letzten drei Jahrzehnten wurde einer Reihe Übersetzungen aus den KHM besondere Aufmerksamkeit gewidmet, die von 1909 bis 1910 in Fortsetzungen unter dem Gesamttitel Shixie (时谐 Zeit-Scherz) in der renommierten Shanghaier Zeitschrift Dongfang zazhi (东方杂志 The Eastern Miscellany) veröffentlicht wurden (Abb. 1). Daher hat Liang 1986 in ihrer Bamberger Dissertation diesen Zeitschriftenabdruck detailliert recherchiert (Liang 1985: 190; Liang 1986: 47-57), ${ }^{1}$ wobei sie allerdings die Buchpublikation, die wenige Jahre nach dem Zeitschriftenabdruck erfolgte, kaum berücksichtigen konnte. Auch von der Vorlage der chinesischen Übersetzung hatte die bisherige Forschung meines Erachtens noch kein Wissen.

Neben den Zeitschriftenabdrucken in der Dongfang zazhi rückte zudem die chinesische Teiltextsammlung von Grimm-Märchen, die wir oben bereits erwähnt haben und von dem Shanghaier Übersetzer Zhou Guisheng ${ }^{2}$ stammt, ins Blickfeld. Es war in der Forschung allerdings lange Zeit sehr umstritten, wie viele der 15 Texte, die Zhou in dem zweiten Band seines Werkes Xin'an xieyi chubian aufgenommen hatte, sich auf die Grimm-Märchen zurückführen lassen. Wu Hongyu konnte 2006 nur sechs Titel aus den KHM nachweisen (vgl. Wu 2006: 66). ${ }^{3}$ Es handle sich nach ihm um Sange xiao airen (三个小矮人 Die drei Männlein), Liang xiongdi (两兄弟 Die zwei Brüder), Lang yu xiaoyang (狼与小羊 Der Wolf und die Geißlein), Xiongpi (熊皮 Der Bärenhäuter), Lang yang fuchou und Yueshi (乐师 Der wunderliche Spielmann). Fu Pinjing stellte dagegen in ihrer Dissertation $2008 \mathrm{zu}$ Recht, allerdings zunächst ohne konkrete Belege und genaue Titelangaben, fest, dass Zhou Guisheng insgesamt zwölf Texte aus den KHM ins Chinesische über-

1 Wegen mangelnden wissenschaftlichen Grundlagenforschungen haben sich zahlreiche spätere chinesische Forscher in den konkreten Publikationsdaten wiederholt geirrt.

2 Zur Biographie von Guisheng Zhou siehe u. a. Yang Shiji (1943: 183-188). Yang würdigte Zhou als den ersten Chinesen, der westliche Literatur in China vorgestellt habe, wusste allerdings nicht, dass Zhou bereits in dessen Textsammlung Xinan xieyi chubian die KHM ins Chinesische übersetzt hatte.

3 Auch eine 2010 fertiggestellte Magisterarbeit behauptete, dass Zhou mindestens fünf Texte aus den KHM übersetzt habe (vgl. Gao 2010: 12). 


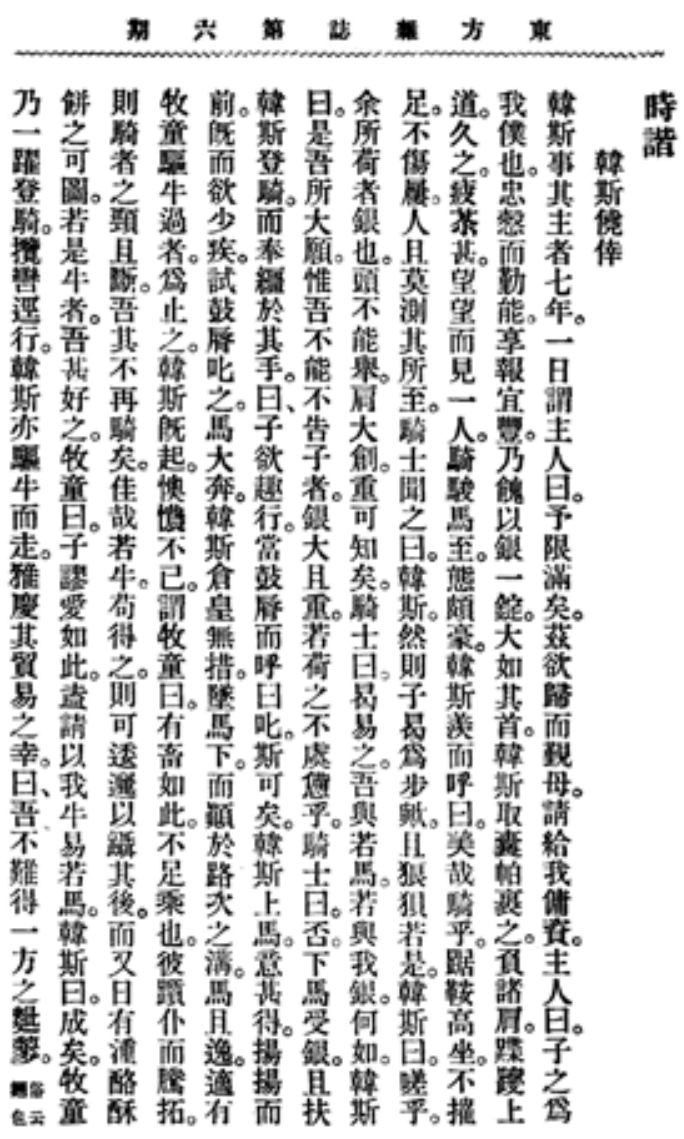

Abb. 1: Hansi jiaoxing (汉斯侥幸 Hans im Glück) als Zeitschriften(vor)abdruck In: Dongfang zazhi 1909 (6). S. 29.

setzt habe (vgl. Fu 2008: 148-152; Fu 2010: 22-23). Sie vertrat die Ansicht, dass Zhou die Texte nicht direkt aus dem Deutschen, sondern entweder aus dem Englischen oder sogar aus dem Französischen übertragen habe.

Es fällt allerdings heute schwer zu ermitteln, welche von den zwölf Texten aus den KHM vorher bereits in der von dem Romancier Li Yuxian, d.i. Li Boyuan (李伯元 1867-1906), im März 1901 in Shanghai gegründeten Literaturzeitung Yuyan bao (寓言报 Fabelzeitung) im Einzelnen abgedruckt wurden. ${ }^{4}$ Laut der Studie von dem renommierten Gelehrten A Ying (阿英) konnten heute allerdings

4 Ohne konkrete Belege behauptete Fu (2010: 177), dass alle diese zwölf Texte aus den KHM bereits in der Zeitung abgedruckt worden seien. 
nur zwei Erstveröffentlichungen von den Märchen Gongzhu (公主 Die Prinzessin) und Xiangren nü (乡人女 Die Bauerntochter), also eigentlich Die drei Schlangenblätter und Die kluge Bauerntochter, in der Zeitung Yuyan bao nachgewiesen werden, und zwar unter der Rubrik Chenji (陈迹 Alte Spuren) (vgl. A 2003: 302; Shi 1986: 221, 241; Wei 1996: 201). ${ }^{5}$ Die zwölf Texte aus den KHM, die im Mai 1903 im zweiten Band von Xin'an xieyi chu bian gesammelt wurden und beim Verlag Qinghua shuju in Shanghai erschienen, ${ }^{6}$ sind heute ohne Weiteres im neunten Band der sämtlichen Werke des namhaften Romanciers Wu Yanren (吴趼人 18661910), der damals die Übersetzungen von Zhou Guisheng redaktionell betreute und herausgab, zugänglich (vgl. Hai 1998: 330-370). Sie lauteten: Mao shu chengqing (猫鼠成亲 Katze und Maus in Gesellschaft; Cat and Mouse in Partnership [KHM $\left.2^{7}\right]$ ), Lang yang fuchou (狼羊复仇 Der Wolf und die sieben jungen Geißlein; The Wolf and the Seven Little Kids [KHM 5]), Yueshi (乐师 Der wunderliche Spielmann; The Wonderful Musician [KHM 8]), Hamo taizi (蛤蟆太子 Der Froschkönig oder der eiserne Heinrich, The Frog Prince [KHM 1]), Lin zhong san ren (林中三人 Die drei Männlein im Walde; The Three Little Men in the Wood [KHM 13]), Shi'er xiongdi (十二兄弟 Die zwölf Brüder; The Twelve Brothers [KHM 9]), Hu shou e yu (狐受鹅愚 Der Fuchs und die Gänse; The Fox and the Geese [KHM 86]), Mouweng (某翁 Der alte Großvater und der Enkel; The Old Grandfather and his Grandson [KHM 78]), Mao yu huli (猫与狐狸 Der Fuchs und die Katze; The Fox and the Cat [KHM 75]), Xiongpi (熊皮 Der Bärenhäuter; Bearskin [KHM 101]), Xiangren nü (乡 人女 Die kluge Bauerntochter; The Peasant's Wise Daughter [KHM 94]) und nicht zuletzt Gongzhu (公主 Die drei Schlangenblätter; The three snake-leaves [KHM 16]).

Neben den 12 Grimm-Märchen enthielt der zweite Band der Sammlung von Zhou Guisheng noch eine prosaische Inhaltsangabe von Shakespeares Drama Kaufmann von Venedig, Yijin ruo (一斤肉 Ein Pfund Fleisch), sowie zwei Fabeln von Äsop, Fou ding wenda (缶鼎问答 The two Pots) ${ }^{8}$ und Lang fu he de (狼负鹤 德 The Wolf and the Crane). Die von der bisherigen Forschung vertretene geläufige Behauptung, dass Xin'an xieyi chubian auch Märchen von Wilhelm Hauff enthalte, ist damit hier zu modifizieren (vgl. Hu 1982: 150-151). Der erste Band der

5 Bei A Ying und Shi Meng wurde der Titel Xiangren nü (乡人女 Bauerntochter) fälschlicherweise als Xiang nüren (乡女人 Bäuerin) angegeben.

6 Zheng Zhiming (2011: 32) konnte anhand einer englischen Ausgabe Grimm's Fairy Tales zehn Texte aus den KHM im zweiten Band der Sammlung Xin'an xieyi chubian identifizieren, wobei er aber zwei weitere, im Konkreten Shi'er xiongdi und Gongzhu, übersah.

7 Die Nummer bezieht sich gewohnheitsmäßig auf die in der 7. Auflage 1857 der Kinder- und Hausmärchen (Grimm 1857) markierte Nummerierung.

8 Einige Forscher haben diesen Titel fäschlicherweise als Ji ding wen da wiedergegeben (vgl. Fu 2010: 22). 
Sammlung von Zhou Guisheng umfasste darüber hinaus noch eine Teilübersetzung des Werkes Tausendundeine Nacht.

Kürzlich haben wir feststellen können, dass einige Texte aus den Kinderund Hausmärchen wie Mao shu chengqin, Yueshi und Mouweng im Verlauf der Zeit noch in der einflussreichen Zeitung Dagong bao (大公报 Ta-Kung-Pao bzw. L'impartial Tientsin), die am 17. Juni 1902 in Tianjin ins Leben gerufen wurde, publiziert wurden. Das Märchen Mao shu chengqin erschien beispielsweise vom 3. Tag des siebten Monats des 29. Regierungsjahres vom Kaiser Guangxu (d.i. 25. August 1903) bis zum 6. Tag des siebten Monats des 29. Regierungsjahres vom Kaiser Guangxu (d.i. 28. August 1903), Yueshi vom 6. Tag des siebten Monats des 29. Regierungsjahres vom Kaiser Guangxu (d.i. 28. August 1903) bis zum 14. Tag des siebten Monats des 29. Regierungsjahres vom Kaiser Guangxu (d.i. 5. September 1903), Mouweng am 17. Tag des siebten Monats des 29. Regierungsjahres vom Kaiser Guangxu (d.i. 8. September 1903) (vgl. Chen 2014: 621, 623, 624, 626; Xie 2008: 171). ${ }^{9}$ Sie wurden alle unter der Rubrik Taixi xiaoshuo (泰西小说 Erzählungen aus dem äußersten Westen) untergebracht.

\section{Popular stories als Textvorlage der chinesischen $\mathrm{KHM}$-Sammlung Shixie}

Bereits in dem Werbetext für die in der Zhicheng shuju (志诚书局 Zhicheng-Buchhandlung) in Beijing lieferbaren Bücher, der am 28. des dritten Monates des ersten Jahres der Regierungszeit Xuantong (d.i. am 17. Mai 1909) in der Zeitung Minhu ribao (民呼日报 Public-Calling Daily) erschien, wurde ein Buch unter dem Titel Shixie angekündigt, versehen mit der Preisangabe von 3 Jiao 5 Fen (三角五分 35 cents) (vgl. Chen 2009: 218). ${ }^{10}$ Nach unserer Studie war das Buch de facto eine ganz andere Publikation, die mit der chinesischen Übersetzung von den KHM der Brüder Grimm in keinerlei Verbindung stand.

Der Umstand, dass die in chinesischer klassischer Schriftsprache angefertigte Märchensammlung Shixie sowohl im Zeitschriften(vor)abdruck als auch in Buchform keinen Originaltitel angab und weder Autor- noch Übersetzernamen nannte, bereitete späteren Forschern große Schwierigkeiten, Originaltitel, Autor

9 Xie wusste allerdings nicht, dass es sich eigentlich um die Übersetzungen von Grimm-Märchen und um die Texte aus der Sammlung Xin'an xieyi chubian handelt.

10 Bei dem heute im Katalog der Universitätsbibliothek der Normal University Beijing verzeichneten 86seitigen Buch, das 1908 von Guanghua bianyi she herauegegeben wurde, handelt es sich mit hoher Wahrscheinlichkeit um ein anderes Buch als die zweibändige KHM-Übersetzung Shixie. 
und Übersetzer zu identifizieren bzw. zu entschlüsseln. Es fiel der Forschung schwer, die richtige Textvorlage, die der chinesischen Textsammlung diente, zu ermitteln. Aufgrund dessen konnte Liang 1986 in ihrer Dissertation nur vermuten, dass 50 unter den 56 Märchen mit Wahrscheinlichkeit Grimm-Märchen waren (vgl. Liang 1986: 45-47).

Was den Übersetzer des Buches Shixie betraf, konnte die bisherige Forschung nur vermuten, wer der Übersetzer gewesen sein könnte. In einem Brief an Rong Zhaozu (容肇祖), der in der Heftnummer 75 der Kantoner Fachzeitschrift Minsu (民俗 Folklore) gedruckt wurde, gab der Forscher Zhang Qingshui (张清水) im August 1929 überraschenderweise den renommierten Übersetzer Lin Qinnan (林琴南) als den eigentlichen Übersetzer von Shixie an (Qing 1929: 61; vgl. Liang 1986: 47, 152). Einige Forscher (z.B. Lu 2011: 84-87; Du 2013: 269) hielten den Buchtitel Shixie fälschlicherweise für den Übersetzernamen.

Nach den Zeitschriftenabdrucken wurde die zweibändige Sammlung Shixie später in Buchform sowohl in die einflussreiche Buchreihe Shuobu congshu (说部丛书 Buchreihe Erzählende Abteilung) als auch in die Buchreihe Xiaoben xiaoshuo (小本小说 Erzählungen in kleiner Buchform) beim Verlag Shangwu yinshuguan (商务印书馆 The Commercial Press) in Shanghai aufgenommen. Die erste Ausgabe in der Buchreihe Shuobu congshu wurde unter Nr. 92 der zweiten Abteilung, also Er ji di jiushi'er bian (二集第九十二编 zweite Abteilung Nr. 92), am 28. Juni 1915 publiziert (Abb. 2). ${ }^{11}$ Die Ausgabe in der Buchreihe Xiaoben xiaoshuo erschien de facto bereits im April 1913. In der Staatsbibliothek Beijing findet sich auch die vierte Ausgabe dieser Buchreihe vom Jahr 1917 in Microfiche-Kopie unter der Signatur MGTS/008476.

Bereits in den 1920er Jahren beachtete der oben bereits erwähnte chinesische Märchenforscher Zhao Jingshen die Märchensammlung Shixie und war bemüht, die originale Textvorlage zu eruieren. Er konnte zunächst nur sehr unkonkret vermuten, dass 60 bis 70 Grimm-Märchen darin ins Chinesische übersetzt worden seien, wobei er die vorausgegangenen Abdrucke in der Zeitschrift Dongfang zazhi nicht wahrnahm. Zhao bemängelte zugleich den für Kinder ungeeigneten gehobenen Sprachstil und die unzutreffende Gattungsangabe von Erzählungen (Zhao 1924 [1922]). Zhao Jingshen modifizierte 1928 teilweise seine Untersuchung und stellte zutreffend fest, dass die chinesische zweibändige Sammlung Shixie umfassend der englischen Textsammlung Grimm's Popular Stories, die bei der Universität

11 Die Registrierung des Buchs unter dem Namen der Brüder Grimm erfolgte bereits 1929 auf einer Liste der ins Chinesische übersetzten Literatur aus Ost- und Westübersee (vgl. Xu 1929: 49). Wei Yixin, der erste chinesische Übersetzer der vollständigen KHM, konnte Shixie als eine Sammlung von Grimm-Märchen identifizieren, wenn er in einer von ihm angefertigten Bibliographie das Buch unter die Namen der Brüder Grimm einordnete (vgl. Wei 1936: 18). 

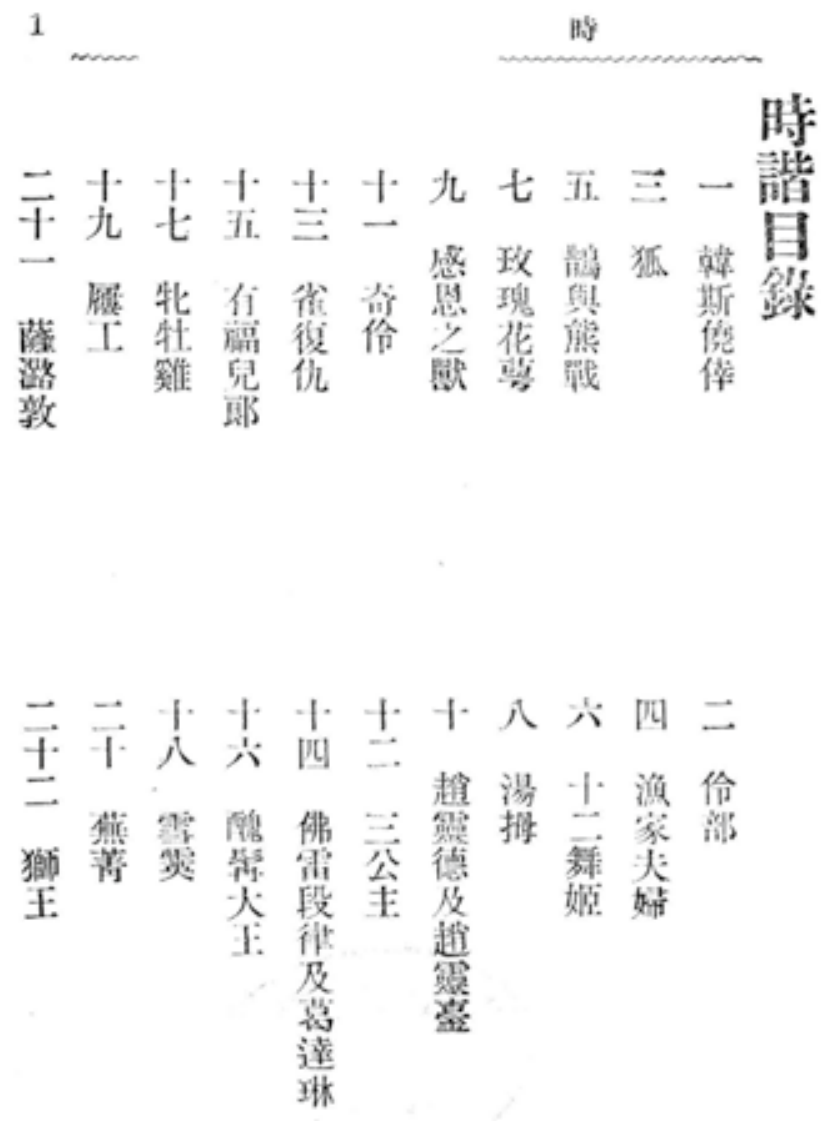

Abb. 2: Inhaltsverzeichnis von Shixie (Grimm 1915: 1)

Oxford erschien, entspräche (Zhao 1928: 14; Zhao 1982 [1928]: 154; vgl. Liang 1986: 47, 152). Leider wurde seine Feststellung lange Zeit von der neueren Forschung übersehen. Bei unserer Untersuchung hat es sich ebenfalls herausgestellt, dass die Reihenfolge der Texte in der chinesischen Sammlung Shixie, in der auch die chinesische Übersetzung von dem Text Peter Klaus the Goatherd, d. h. einem Text von Othma, d.i. Johann Karl Christoph Nachtigall (1753-1819), auftauchte, umfassend mit der Reihenfolge der englischen Grimm-Ausgaben von 1868 bzw. 1875 übereinstimmte (Grimm 1868: 233-237; Grimm 1875). Genauer betrachtet stimmten die chinesischen Übersetzungen und die Reihenfolge der Texte in Shixie sowohl mit der zweibändigen Ausgabe German popular stories (Grimm 1823-1826; Grimm 1904 [1823-1826]; Grimm 1905 [1823-1826]; Grimm 1910 [1823-1826]; Grimm 1915 [1823-1826]) als auch mit der englischen Grimm-Ausgabe German popular stories 


\section{CONTENTS.}

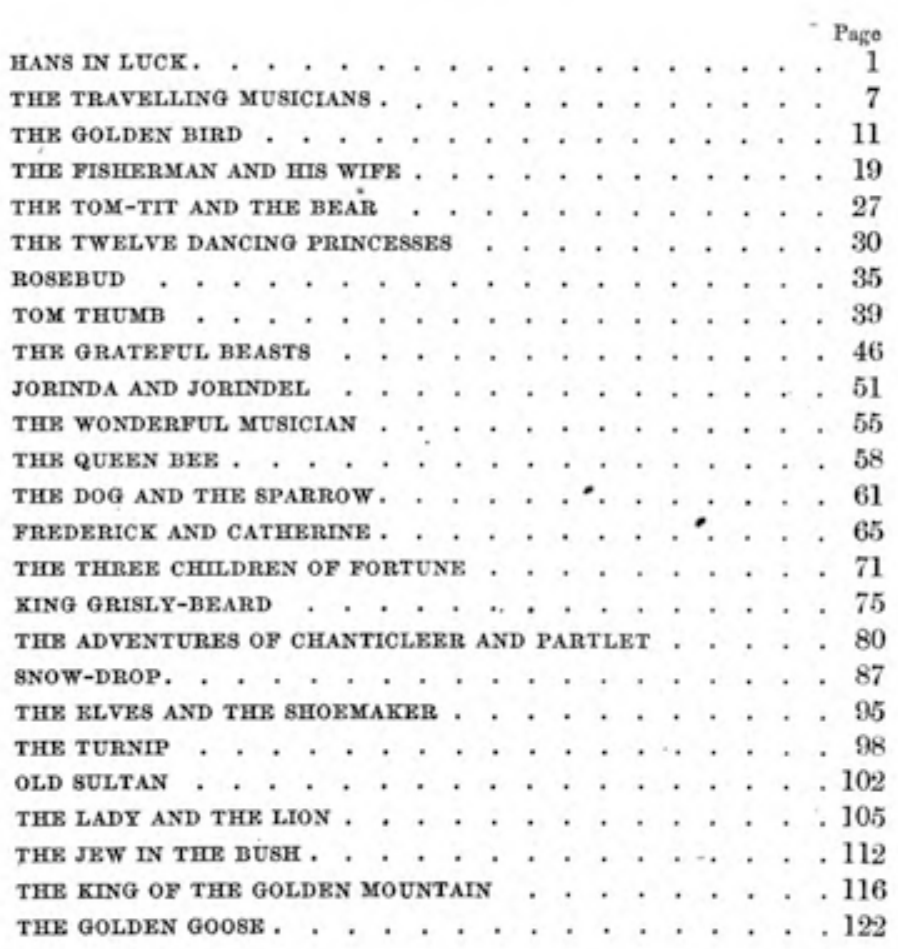

Abb. 3: Inhaltsverzeichnis von German popular stories (Grimm 1868: xxv)

with illustrations after the designs of George Gruikshank (Grimm 1868; Grimm 1875; Grimm 1913) (Abb. 3) überein. Zhao Jingshen irrte sich allerdings im Buchtitel der originalen Vorlage und gab darüber hinaus keinen konkreten Publikationsort bzw. kein Erscheinungsjahr an, als er 1928 in einem Artikel konstatierte: Die in der Buchreihe Shuobu congshu publizierte zweibändige Shixie sei

völlig nach der Ausgabe der University of Oxford Gelin tonghua ji (格林童话集 Ausgewählte Grimm-Märchen) übersetzt worden. Sie umfasste 56 Texte, einen Text mehr als das Originalbuch, weil der 36. und der 37. Text in Shixie eigentlich ein einziger Text im Originalbuch waren. Shixie hat diesen Text in zwei Teile aufgeteilt und deshalb ist ein Text mehr darin enthalten. Das Originalbuch heißt Grimm's Popular Stories, versehen mit 22 sehr kostbaren Illustrationen von Gruikshank, aus denen Herr Zhou Zuoren in seinem Buch Ziji de yuandi (自己的园地 Mein eigenes Gartenfeld) das Bild The elves and the shoemaker wiederabgedruckt hat. (Zhao 1982 [1928]: 154) 


\section{POPULAR STORIES.}

\section{HANS IN LUCK.}

HANS had served his master seven years, and at last said to him, "Master, my time is up, I should like to go home and see my mother; so give me my wages." And the master said, "You have been a faithful and good servant, so your pay shall be handsome." Then he gave him a piece of silver that was as big as his head.

Hans took out his pocket-handkerchief, put the piece of silver into it, threw it over his shoulder, and jogged off homewards. As he went lazily on, dragging one foot after another, a man came in sight, trotting along gaily on a capital horse. "Ah!" said Hans aloud, "what a fine thing it is to ride on horseback! there he sits as if he was at home in his chair; he trips against no stones, spares his shoes, and yet gets on he hardly knows how." The horseman heard this, and said, s

Abb. 4: Hans in Luck (Grimm 1910 [1823-1826]: 1)

Die von Zhao erwähnte Grimm-Ausgabe der University of Oxford ist die 1904 in Oxford erschienene zweibändige Ausgabe German popular stories (Grimm 1904 [1823-1826]). Zhaos Feststellung stimmte nicht gänzlich, wenn wir den Umstand berücksichtigen, dass der zweite Band der chinesischen Sammlung Shixie mit dem Text Qingdeng (青灯 The blue light) begann, während der zweite Band der 1904 in Oxford faksimilierten Ausgabe mit dem Text The Goose-Girl begann. Das heißt, die chinesische Übersetzung bediente sich eher einer einbändigen als der von Zhao vermuteten zweibändigen Ausgabe. Trotzdem können wir nun festhalten, dass sich die chinesische Übersetzung von Grimm-Märchen Shixie de facto der englischen Textvorlage bediente. Aufgrund der Offenlegung der englischen Textvorlage ist der chinesische Titel Shixie zum ersten Mal nachvollziehbarer zu verstehen: Er bezieht sich eigentlich auf die Übertragung des englischen Titels 
Popular stories (Abb. 4) ins Chinesische: Shixie (时谐 Zeit-Scherz, also in der Gegenwart beliebte scherzhafte Geschichten).

\section{Neu ermittelte Befunde und Rezeptionsbelege in Zeitschriften und Zeitungen im zweiten Jahrzehnt des 20. Jahrhunderts}

Es wurden inzwischen Dutzende neue, von der bisherigen Forschung kaum beachtete, frühere Einzelübertragungen der Grimm-Märchen recherchiert, die verstreut in den Zeitungen wie Shenbao (申报 Shun Pao) und Zeitschriften wie Xingqi xiaoshuo (星期小说 Wochenerzählungen), Zhonghua xiaoshuo jie (中华小 说界 ChungHua Novel Magazine), Libai liu (礼拜六 Samstag), Shanghai tang (上 海滩 Der Shanghaier Bund) u. a. erschienen.

Unter anderem publizierte die berühmteste Shanghaier Zeitung Shenbao vom 28. des zehnten Monats bis zum 1. des elften Monats des dritten Jahres vom Kaiser Xuantong (d.i. vom 18. bis 20. Dezember 1911) unter der Rubrik Ziyou tan (自由谈 Freie Plaudereien) eine chinesische Übertragung Jigong hua (棘宫花 Dornröschen [KHM 50]) in drei Fortsetzungen. Der Übersetzer bezeichnete sich als Zhongguo Yemin (中国野民 Ein chinesischer Wilder). Bemerkenswerterweise wurde der eigentliche Autor des Textes als Deguo Geleimei (德国格雷美 Grimm aus Deutschland) angegeben, wobei das Genre als Duanpian shenguai (短篇神 怪 Kleine Geistergeschichte) charakterisiert wurde (vgl. Liu 2008; Kan 2013: 87, 89, 289).

Ebenfalls im Jahr 1911 erschien in der literarischen Wochenschrift Xingqi xiaoshuo (Heft 87) in Tianjn eine siebenseitige Übersetzung von Muqin Haoluo (母亲豪罗 Frau Holle [KHM 124]) unter der Rubrik Duanpian xiaoshuo (短篇小说 Kleine Erzählung). Es fand sich ein wortgetreuer Wiederabdruck in der Beilage der Beijinger Zeitung Chenbao (晨报 Morgenzeitung) vom 4. April 1923 (Nr. 84, S. 3) mit der Übersetzerangabe von Zhong Chen (仲宸), und zwar Wang Zhongchen (王仲宸), unter der Rubrik Xiaoshuo (小说 Erzählung). ${ }^{12}$

Die chinesische Übersetzung Jingbian de yang'e'nü (井边的养鹅女 Die Gänsehirtin am Brunnen [KHM 179]), die von Cui An (崔莽) ${ }^{13}$ angefertigt wurde,

12 Der Verfasser verfügt über einen Textbeleg in digitaler Form.

13 Cui An, der offenkundig seine Übersetzungen anhand der deutschen Vorlagen anfertigte, publizierte Ende 1914 im Heft 4 der Zeitschrift Shanghai tang noch die Übersetzung Shuta (鼠塔 Mäuseturm) von den Brüdern Grimm (S. 30-33). 


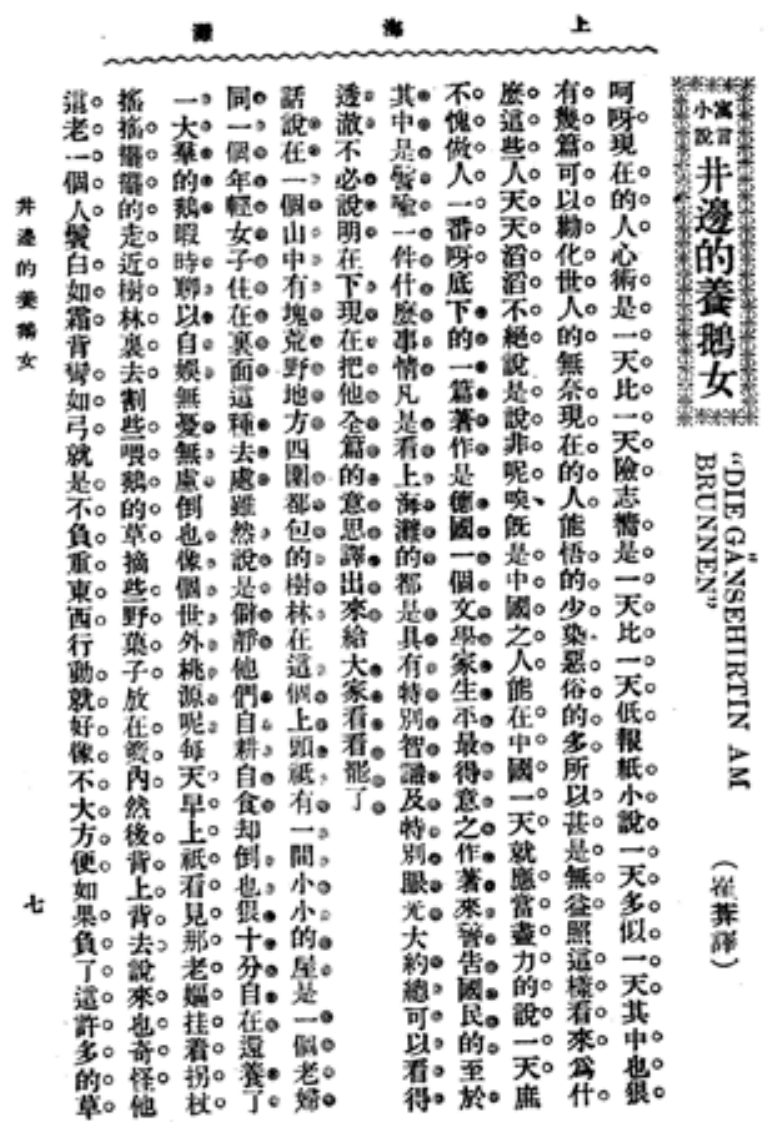

Abb. 5: Die von Cui An (崔蓄) unmittelbar aus dem Deutschen angefertigte Übersetzung Jingbian de yang'e'nü (井边的养鹅女 Die Gänsehirtin am Brunnen [KHM 179]) In: Shanghai tang (上海滩 Der Shanghaier Bund) 1915 (5). S. 7-20.

erschien am 29. Januar 1915 in der Literaturzeitschrift Shanghai tang (Abb. 5), die im Dezember 1914 in Shanghai ins Leben gerufen wurde und dreimal im Monat erschien, und zwar mit der Genreangabe Yuyan xiaoshuo (寓言小说 Fabel-Erzählung) (Heft 5, S. 7-20). ${ }^{14}$

In der populären literarischen Wochenschrift Libai liu, die auch den englischen Titel The Saturday trug, die im Juni 1914 in Shanghai ins Leben gerufen wurde, erschienen 1915 ein paar Texte, die sich als Grimm-Märchen identifizieren

14 Nachgewiesen in Zhou (1991: 277), Wei (1996: 68) und Liu (2008: 144). Eine Faksimile-Kopie liegt bei dem Verfasser. 
lassen: San jinfa (三金发 Der Teufel mit den drei goldenen Haaren [KHM 29]) von Ai Lu (爱庐) (Heft 41 vom 13. März 1915), Jinwan yuan (金丸缘 Der Froschkönig oder der eiserne Heinrich [KHM 1]) ebenfalls von Ai Lu (Heft 42 vom 20. März 1915) und Wanneng yisheng (万能医生 Doktor Allwissend [KHM 98]), übersetzt von Xiao Cao (小草) (Heft 44 vom 3. April 1915, S. 16-21), mit Angabe vom Autorennamen Kelimu (克利姆 Grimm).

In der zweiten Hälfte vom Februar 1915 veröffentlichte Zhuang Hui (壮悔), d.i. Li Zhuanghui (李壮悔), Li Sichun (李思纯 1893-1960), in der populären Halbmonatsschrift Yuxian lu (娱闲录 Entertainment Record) (Nr. 15, S. 14-16), die als Beilage von der Zeitung Sichuan gongbao (四川公报 Sichuan Bulletin Supplement) im Juli 1914 in Chengdu gegründet wurde, die Übersetzung Shishi meiren (石室美人 Schöne Frau in der Grotte) mit der Genrebezeichnung Shenguai xiaoshuo (神怪小说 Geistergeschichte). Es handelte sich eigentlich um die Übersetzung von Das Wasser des Lebens [KHM 97].

Im August 1916 veröffentlichte die populäre Shanghaier Monatsschrift Funü shibao (妇女时报 Women's Times) ${ }^{15}$ die von Cui An und Yan Qiu (雁秋) gemeinsam angefertigte Übersetzung Baixue gongzhu yu qi airen (白雪公主与七矮人 Die Schneewittchen und die sieben Zwerge) (Nr. 19, S. 89-100). Bemerkenswerterweise wurde der Autor als Deguo wenxue dajia Ke lin (德国文学大家克林 der große deutsche Dichter Grimm) bezeichnet, wobei die Gattung als Shenhua xiaoshuo (神话小说 Mythologische Erzählung) charakterisiert wurde.

Im Mai 1917 publizierte die Shanghaier populäre Monatsschrift Yuxing (余兴 The Magazine Eastern Times) in der Heftnummer 28 die von Ting Wu (听梧) angefertigte Übersetzung Shuang meigui (双玫瑰 Doppelrosen), die unter der Genrebezeichnung Shenguai xiaoshuo (神怪小说 Geistergeschichte) erschien und sich als Schneeweißchen und Rosenrot [KHM 161] identifizieren lässt. Die Übersetzung war bereits vorher vom 29. Juni bis zum 1. Juli 1916 in Shibao (时报 The Eastern Times) in Fortsetzungen abgedruckt worden.

Im dritten Jahrgang der literarischen Monatsschrift Zhonghua xiaoshuo jie, die im Januar 1914 in Shanghai ins Leben gerufen wurde, erschienen 1916 mehrere von Chen Jialin (陈家麟) und Chen Dadeng (陈大镫) angefertigte Übersetzungen von Grimm-Märchen, die meistens mit der Genrebezeichnung Shenguai xiaoshuo versehen wurden: Shuishen (水神 Die Nixe im Teich [KHM 181]) (veröffentlicht am 1. Januar 1916) (Abb. 6), Nielong qishe ji (蕉龙七舌记 Die zwei Brüder) (veröffentlicht am 1. Februar 1916), Wuya gongzu (乌鸦公主 Die Rabe [KHM 93]) (veröffentlicht am 1. März 1916), Shiguo qiyuan (失果奇缘 Der goldene Vogel [KHM 57]) (ver-

15 Bei Chen Mingshu (1994: 422) wurde die Zeitschrift fälschlicherweise als Funü zazhi (妇女杂 志 Ladies' Journal) angegeben. 


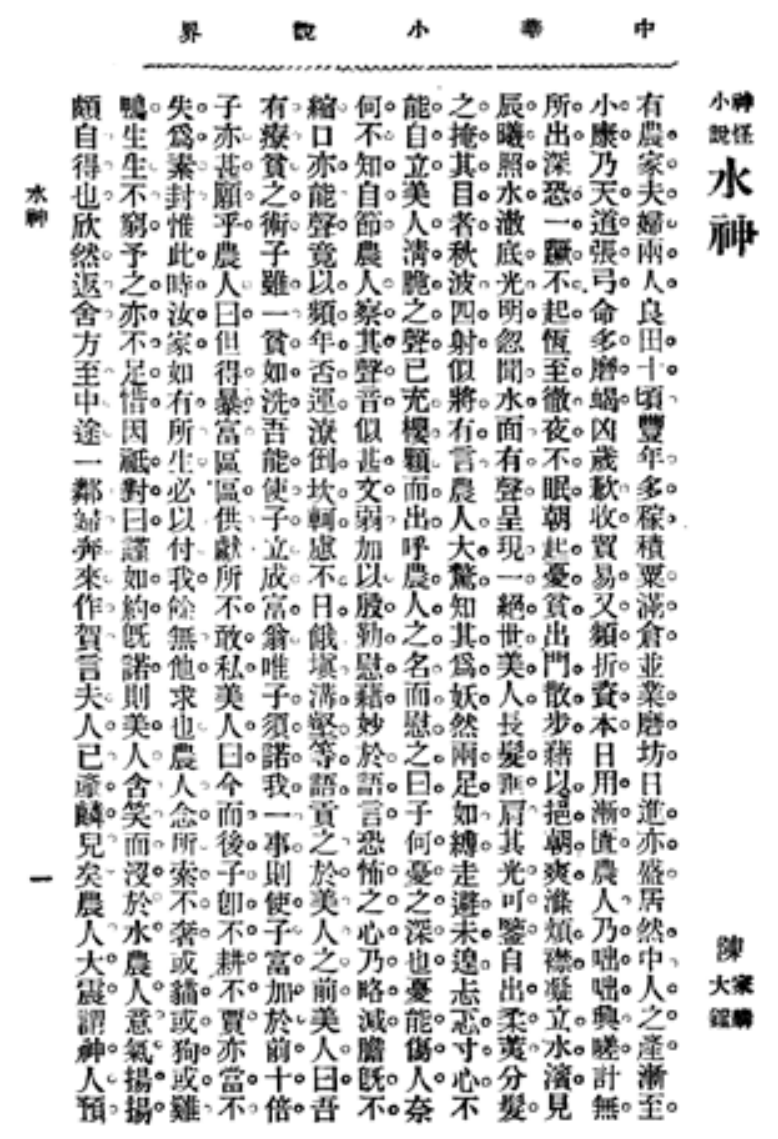

Abb. 6: Die von Chen Jialin (陈家麟) und Chen Dadeng (陈大镫) angefertigte Übersetzung Shuishen (水神 Die Nixe im Teich [KHM 181]) In: Zhonghua xiaoshuo jie (中华小说界 ChungHua Novel Magazine) 3 (1). Veröffentlicht am 1. Januar 1916.

öffentlicht am 1. April 1916), E wei qi ren (鹅尾七人 Die goldene Gans [KHM 64]), die mit der Gattungsbezeichnung Huji xiaoshuo (滑稽小说 komische Erzählung) (veröffentlicht am 1. Mai 1916) versehen wurde, ${ }^{16}$ und nicht zuletzt Ya yan (鸦言 Die drei Raben) (veröffentlicht am 1. Juni 1916), dessen Originaltext zwar nicht in den üblichen KHM-Ausgaben, aber in einer englischen Grimm-Übersetzung nachgewiesen worden ist (vgl. Grimm 1901: 202-206).

16 Auch vom 20. bis 22. November 1919 erschien eine Erzählung von Yu Bing (玉冰) unter dem Titel Ewei qi ren (我鸟尾七人 Die goldene Gans [KHM 64]) in der Zeitung Yuandong bao (远东报 Zeitung des Fernen Ostens). 
In der Shanghaier Zeitung Shibao erschien vom 23. August 1916 die von Bi Wu (碧梧), d.i. Zhang Biwu (张碧梧), geboren 1897, angefertigte Übersetzung Yanling shui (延龄水 Das Wasser des Lebens [KHM 97]). Beachtenswerterweise wurde die Gernebezeichnung Taixi tonghua (泰西童话 Märchen aus dem äußerst Westen) benutzt (vgl. Liu 2011: 339). Ebenfalls in der Zeitung Shibao publizierte Chen Zhaoyuan (陈肇援) vom 26. bis 27. September 1917 die Übersetzung San jinfa (三金发 Der Teufel mit den drei goldenen Haaren [KHM 29]) mit Angabe des englischen Titels The Three Golden Hairs in Fortsetzungen (vgl. Liu 2011: 345).

Die Übersetzung Han Sui'er yu Zha Laitui'er xiongmei beinan ji (汉绥尔与轧 莱退尔兄妹被难记 Hänsel und Gretel [KHM 15]), die gemäß der vom Übersetzer unter der Überschrift angegebenen Information direkt aus dem Deutschen ins Chinesische übertragen werden sollte, erschien vom 8. bis 10. November 1917 in Fortsetzungen ebenfalls in der Shanghaier Zeitung Shibao. Der Übersetzer trug den Namen (?) Hu diao zhe (?湖钓者 Fischer am See?). ${ }^{17}$ Sie wurde auch vom 28. bis 30. März 1918 in der populären Pekinger Zeitung Chenzhong bao (晨钟报 Morning Bell Daily) wieder abgedruckt (vgl. Liu 2011: 491).

\section{Fazit}

Die frühe Rezeption der Kinder- und Hausmärchen der Brüder Grimm fällt in das letzte Jahrzehnt der Qing-Dynastie und dauerte bis zur 4. Mai-Bewegung 1919. Sie zeichnet sich, wie unsere Studie gezeigt hat, durch eine markante und komplexe Anonymität aus. Trotz der Ausblendung von Autoren- und unter Umständen zumeist auch von Übersetzernamen sowie verwirrenden Variationen bei Angabe der Gattungsbezeichnung Märchen war es doch möglich, im Sinne der Spurensuche einschlägige Übersetzungs- und Rezeptionsbelege $\mathrm{zu}$ identifizieren. Mittels neu recherchierter Befunde haben wir den Versuch unternommen, die Desiderate in der bisherigen Forschung zu beseitigen, wobei wir ein besonderes Augenmerk auf die in der bisherigen Forschung kaum beachtete originale Vorlage der ersten chinesischen KHM-Sammlung Shixie sowie auf die zahlreichen in Zeitschriften und Zeitungen zerstreut publizierten Belegtexte gerichtet haben.

Die hier präsentierten Forschungsergebnisse sind im Rahmen eines staatlich geförderten Projektes zur Erschließung von entlegenen Übersetzungs- und Rezeptionsbelegen der deutschsprachigen Literatur in der Zeit der Spät-QingDynastie entstanden. Die Texte, deren Autorennamen in den meisten Fällen vorsätzlich oder gewohnheitsmäßig verschwiegen wurden und zerstreut in Zeit-

17 Das erste Zeichen des Übersetzernamens ist leider momentan noch nicht zu eruieren. 
schriften, Zeitungen und Bücherreihen vorlagen, weisen eine aktive Rezeptionsund Übersetzungstätigkeit nach, die betreffend der Kinder- und Hausmärchen der Brüder Grimm in der unruhigen chinesischen Umbruchszeit erfolgte, und zeugen sowohl von einem breiten und vielfältigen Spektrum von der Adaption des fremdkulturellen Gedankenguts als auch von einer ungewöhnlichen Intensivität von Bemühungen des chinesischen Kulturkreises, im Zeichen der Modernisierungsund Erneuerungsprozesse interkulturelle Dialoge mit den fremden Kulturen zu führen.

\section{Literaturverzeichnis}

A, Ying (阿英). 2003. Aufzeichnungen über die kleinen Zeitungen in der Spät-Qing-Dynastie (晚清小报录). In: A, Ying (阿英). Gesammelte Werke von A Ying. Band 6 (《阿英全集》 第六卷). Hefei: Anhui Education Press (安徽教育出版社). S. 273-322.

Chen, Dakang (陈大康). 2009. Public-Calling Daily in the late Qing Dynasty and Correlative Chronicle of Novels (《民呼日报》与小说有关编年). In: The Journal of Ming-Qing Fiction studies (《明清小说研究》) (2). S. 215-226.

Chen, Dakang (陈大康). 2014. Eine chronologische Geschichte der chinesischen Erzählungen in der Neuzeit. Band 2 (《中国近代小说编年史》第二卷). Beijing: People’s Literature Publishing House (人民文学出版社).

Chen, Mingshu (陈鸣树) (Hrsg.). 1994. Eine chronologische Bibliographie von der chinesischen Literatur im 20. Jahrhundert (1897-1929) (《二十世纪中国文学大典（1897-1929）》). Shanghai: Shanghai Education Press (上海教育出版社).

Du, Rong. 2013. Rezeption der Kinder- und Hausmärchen der Brüder Grimm in China. In: Literaturstraße 14. S. 267-280.

Fu, Pinjing (付品晶). 2008. Verbreitung und Wandlung der chinesischen Übersetzung von Grimms Märchen (格林童话汉译流传及变异). In: Journal of Southwest Minzu University (Humanities and Social Science) (《西南民族大学学报 (人文社科版)》) 29 (2). S. 148-150.

Fu, Pinjing (付品晶). 2010. Grimms Märchen in China (《格林童话在中国》). Chengdu: Sichuan Literature and Art Publishing House (四川文艺出版社).

Gao, Qian (高倩). 2010. German Fairy Tales in China. The Translation, Spread, Accept and Influence of Grimm Fairy Tales in China (《德语童话的中国漫游一一以格林童话为代表 谈其在中国的译介、传播、接受和影响》). Shanghai: East China Normal University. Manuskript von Magisterarbeit.

Grimm, Wilhelm/Grimm, Jacob. 1823-1826. German Popular Stories. Translated from the Kinder- und Hausmärchen. Collected by M. M. Grimm, From Oral Tradition; [Illustrator: George Cruikshank]. In two series. London: C. Baldwyn.

Grimm, Wilhelm/Grimm, Jacob. 1857 [1837]. Kinder- und Hausmärchen. Gesammelt durch die Brüder Grimm. 2 Bände. Große Ausgabe. 7. Auflage. Göttingen: Verlag der Dietrichschen Buchhandlung.

Grimm, Wilhelm/Grimm, Jacob. 1868. German Popular Stories with Illustrations after the Original Designs of George Cruikshank. In two series. Edited by Edgar Taylor, with introduction by John Ruskin. M. A. London: Hotten. 
Grimm, Wilhelm/Grimm, Jacob. 1875. German Popular Stories: with Illustrations after the Original Designs of George Cruikshank. Edited by Edgar Taylor; with introduction by John Ruskin. London: Chatto and Windus.

Grimm, Wilhelm/Grimm, Jacob. 1901. German Popular Stories and Fairy Tales. As told Gammer Grethel. From the collection of. M. M. Grimm. Rev. translation by Edgar Taylor. With illustrations from designs by George Cruikshank and Ludwig Grimm. London: George Bell and Sons.

Grimm, Wilhelm/Grimm, Jacob. 1904 [1823-1826]. German popular stories. [tr. and ed. by E. Taylor] from the Kinder- und Hausmärchen, collected by m. m. Grimm. 2 Vols. [Facs. repr. of the 1st ed.]. [Oxford: Oxford University Press].

Grimm, Wilhelm/Grimm, Jacob. 1905 [1823-1826]. Popular Stories. Collected by the brothers [d. i. Jacob und Wilhelm] Grimm. With twenty-two illustrations by George Cruikshank. A reprint of the first English edition; Oxford edition. London u. a.: Henry Frowde.

Grimm, Wilhelm/Grimm, Jacob. 1910 [1823-1826]. Popular Stories. Collected by the brothers [d. i. Jacob und Wilhelm] Grimm. With twenty-two illustrations by George Cruikshank. A reprint of the first English edition; Oxford edition. London u. a.: Henry Frowde Oxford University Press.

Grimm, Wilhelm/Grimm, Jacob. 1913. German Popular Stories with Illustrations after the Designs of George Gruikshan. Edited by Edgar Taylor with an introduction by John Ruskin. London: Chatto and Windus.

Grimm, Wilhelm/Grimm, Jacob. 1915. Shixie (《时谐》). Band 1. Shanghai: The Commercial Press (商务印书馆).

Grimm, Wilhelm/Grimm, Jacob. 1915 [1823-1826]. Popular Stories. Coll. by the brothers [d. i. Jacob und Wilhelm] Grimm. With twenty-two illustrations by George Cruikshank. A reprint of the first English edition; Oxford edition. London u. a.: Oxford University Press.

Hai, Feng (海风) (Hrsg.). 1998. Sämtliche Werke von Wu Yanren. Band 9. (《吴趼人全集第九 卷》). Ha'erbin: Beifang Literatur and Art Publishing House (北方文艺出版社).

$\mathrm{Hu}$, Congjing (胡从经). 1982. Zhou Guishengs Übersetzungen von der Kinderliteratur (周桂笙的 儿童文学翻译作品). In: Hu, Congjing. 1982. Quellensuche nach der verschollenen Kinderliteratur in der Spätqing-Dynastie (《晚清儿童文学钩沉》). Shanghai: Children Publishing House (少年儿童出版社). S. 148-159.

Kan, Wenwen (阙文文). 2013. Die in den Zeitschriften und Zeitungen der Spätqing-Dynastie publizierten übersetzten Erzählwerke (《晚清报刊上的翻译小说》). Jinan: Shangdong Qilu Press Co. (齐鲁书社).

$\mathrm{Li}$, Dechao. 2014. The Influence of the Grimms' Fairy Tales on the Folk Literature Movement in China (1918-1943). In: Joosen, Vanessa/Lathey, Gillian (Hrsg.): Grimms' Tales around the Globe. The Dynamics of Their International Reception. Detroit: Wayne State University Press. S. 119-133.

Liang, Yea-Jen. 1985. Kennen Chinesen die Märchen der Brüder Grimm? In: Denecke, Ludwig (Hrsg.): Brüder Grimm Gedenken. Band 5. Marburg: Elwert. S. 176-191.

Liang, Yea-Jen. 1986. Kinder- und Hausmärchen der Brüder Grimm in China: Rezeption und Wirkung. Wiesbaden: Otto Harrassowitz.

Liu, Yongwen (刘永文). 2008. Bibliographie von den Erzählwerken in der Spätqing-Dynastie (《晚清小说目录》). Shanghai: Shanghai Classics Publishing House (上海古籍出版社).

Liu, Yongwen (刘永文). 2011. Bibliographie von den im Zeitraum von 1912 bis 1920 erschienenen Erzählwerken (《民国小说目录 (1912-1920)》). Shanghai: Shanghai Classics Publishing House (上海古籍出版社). 
Lu, Xia. 2011. Grimms Märchen in China: Begegnung, Rezeption und Wirkung. Aachen: ShakerVerlag.

Lu, Xia (陆霞). 2012. Grimms Märchen in China: Begegnung, Rezeption und Wirkung (《走进格 林童话诞生接受价值研究》). Chengdu: Sichuan Literature and Art Publishing House (四川 文艺出版社).

Qing, Shui (清水). 1929. Briefwechsel mit der Redaktion (Brief an Herrn Rong Zhaozu) (本刊通讯: 致容肇祖先生). In: Folklore (《民俗》)(75). Veröffentlicht am 28. August 1929. S. 59-62.

Shi, Meng (时萌). 1986. Zhou Guisheng und Xu Nianci (《周桂笙与徐念慈》). In: Shi, Meng. 1986. Beiträge über die chinesische Literatur in der Neuzeit (《中国近代文学论稿》). Shanghai: Shanghai Classics Publishing House (上海古籍出版社). S. 219-262.

Sun, Jianjiang (孙建江). 2013. Einführung in die chinesische Kinderliteratur im 20. Jahrhundert (《二十世纪中国儿童文学导论》). Chengdu: Sichuan Publishing Co. (四川出版集团).

Wei, Shaochang (魏绍昌) (Hrsg.). 1996. Die große Sammlung von der chinesischen Literatur in der Neuzeit. Abteilung: Bibliographien und Register. Band 2. (《中国近代文学大系史料索 引集 第二卷》). Shanghai: Shanghai Bookstore (上海书店).

Wei, Yixin (We I-Hsin魏以新). 1936. Liste der deutschen Bücher, die bisher ins Chinesische übersetzt worden sind (《中译德文书籍目录》). Woosung: [0. Verl.].

Wu, Hongyu (伍红玉). 2006. Die Evolution der Versionen der Grimmschen Märchen und ihre chinesische Übersetzung in der Neuzeit (格林童话的版本演变及其近代中译). In: Deutschland-Studien (《德国研究》) 21(4). S. 62-68.

Wu, Hongyu (伍红玉). 2010. Geschichten hinter dem Märchen. Die westlichen Märchen und die chinesische Gesellschaft (1900-1937)（《童话背后的历史 - 西方童话与中国社会 (1900-1937) 》). Taipei: Taiwan xuesheng shuju (台湾学生书局).

Xie, Renmin (谢仁敏) .2008. „Ta Kung bao“ Novel and Novel Concept in Ying Lianzhi period (英玫之时期的《大公报》小说及其小说观念). In: Jiang-huai Tribune (《江淮论坛》) (5). S. 171-176.

Xu, Bai (虚白). 1929. Katalog von den aus dem Ost- und Westübersee ins Chinesische übersetzten literarischen Werken (《汉译东西洋文学作品编目》). Shanghai: Truth, Beauty, and Goodness (真美善).

Yang, Shiji (杨世骥). 1943. Aufzeichnungen über Zhou Guisheng (记周桂笙). In: New Chung Hwa (The republished number) (《新中华》复刊号). 1 (1). S. 183-188.

Zhao, Jingshen (赵景深). 1924[1922]. Kurze Biographie von den Märchendichtern den Brüdern Grimm (童话家格林弟兄传略). In: Zhao, Jingshen (赵景深). (Hrsg.): Interpretationen von Märchen (《童话评论》). Shanghai: Xinwenhua shushe (新文化书社). S. 177-188. Ursprünglich unter dem Titel: Kurze Biographie von den pädagogischen Märchendichtern den Brüdern Grimm (教育童话家格林弟兄传略). In: Chenbao fukan (《晨报副刊》). 26. Mai 1922. S. 1-2; 27. Mai 1922. S. 1-2.

Zhao, Jingshen (赵景深). 1982 [1928]. Rezension von „Some types of Indo-European folktale“ (评《印欧民间故事型式表》). In: Zhao, Jingshen (赵景深). 1982. Beiträge zur volkstümlichen Literatur (《民间文学丛谈》). Changsha: Hunan People Press (湖南人民出 版社). S. 154-157. Ursprünglich in: Zhao, Jingshen (赵景深). 1928. Studien zu Volkssagen (《民间故事研究》). Shanghai: Fudan shudian (复旦书店). S. 13-18.

Zheng, Zhiming (郑世明). 2011. Rediscovering the Value of Humorous Translated Stories by Xin'an in Translation History (《新庵谐译初编》翻译史价值再发现). In: Journal of Hebei North University (Social Science Edition) (《河北北方学院学报 (社会科学版)》) 27(5). S. 30-33. 


\section{Angaben zur Person}

Prof. Dr. Xiaoqiao Wu, Direktor der Department of German an der Beihang University in Beijing. Seine Forschungsschwerpunkte sind neuere deutsche Literaturwissenschaft (Weimarer Klassik, Romantik, Poetischer Realismus und Klassische Moderne), deutsch-chinesische Literatur- und Kulturbeziehungen, Komparatistik und Transferforschung. 